$16^{\text {th }}$ International Conference on

AEROSPACE SCIENCES \& AVIATION TECHNOLOGY,

$\boldsymbol{A S A T}$ - 16 - May 26 - 28, 2015, E-Mail: asat@mtc.edu.eg

Military Technical College, Kobry Elkobbah, Cairo, Egypt

Tel : +(202) 24025292 - 24036138, Fax: +(202) 22621908

\title{
Improving the In-plane Stiffness of the Hexagonal Re-entrant Auxetic Honeycomb Cores
}

\author{
M. Othman ${ }^{*}$, K. Zied ${ }^{\dagger}$, T. Elmahdy
}

\begin{abstract}
In the present work, two new designs of honeycomb structures based on the basic re-entrant structure were presented namely, splined-reentrant honeycomb and stiffenedreentrant honeycomb. The new structures were designed in order to improve the in-plane stiffness of the honeycomb and keeping the auxetic behaviour of the structure. In this study, finite elements modelling and experimental work were carried out to evaluate the in-plane mechanical properties of the new designs. The effect of the geometrical parameters such as rib length, rib thickness and orientation of the unit-cell of the new designs on the in-plane properties was investigated. Finite element results were compared with the analytical results to verify the finite element models of the basic re-entrant structure and good agreement was obtained. The finite element results showed that the in-plane stiffness of the new designs was improved significantly compared to the basic re-entrant structure. Also, the stiffened-reentrant structure showed better enhancement of the in-plane stiffness than the splined-reentrant structure. However the auxeticity of the splined-reentrant structure is higher than the stiffened-reentrant structure. For example the modulus of elasticity of the stiffened-reentrant structure 10 times that of the basic reentrant structure in $\mathrm{x}$-direction and over two times in the $y$-direction with lower values of the negative Poisson's ratio. Compression tests were carried on honeycomb samples made of steel using laser cutting technique with different geometrical parameters. Test results for the three designs were compared with the finite element results and they were in a good agreement.
\end{abstract}

Keywords: Honeycombs, Auxetic, negative Poisson's ratio, In-plane properties, re-entrant.

\section{Nomenclature}

$\begin{array}{ll}\text { Symbol } & \text { Meaning } \\ b & \text { The auxetic structure depth } \\ E_{s} & \text { Young's modulus of the solid material of the structure } \\ E_{x} & \text { Young's modulus in x-direction of the auxetic network } \\ E_{v} & \text { Young's modulus in x-direction of the auxetic network } \\ h & \text { Ligament height for unequal rib re-enterent hexagonal cell } \\ l & \text { Ligament length } \\ t & \text { rib thickness } \\ \theta & \text { The rib orientation } \\ v_{x y}, v_{y x} & \text { In-plane Poisson's ratio of the tructure }\end{array}$

\footnotetext{
* Assistant Lecturer, Faculty of Engineering, Mataria, Helwan University, Cairo, a_osman9@yahoo.com $\dagger$ Assistant Professor, Faculty of Engineering, Mataria, Helwan University, Cairo, kmzied@ hotmail.com

* Associated Professor, Faculty of Engineering, Mataria, Helwan University, Cairo, tmelmahdy@gmail.com
} 


\section{Introduction}

There is a growing interest in petroleum, construction, automotive, aerospace industries to use new materials to reduce weight of systems components for better performance, reducing fuel consumption, exhaust emission and noise reduction. Advanced materials such as honeycomb structures come to light as a feasible candidate in these fields. This type of structures can be used in cabs, floors, walls, doors, panels and roofs for vans, trucks, trailers trains and airplanes. The honeycombs and their sandwich panels find interest also in state-of the-art new buildings as noise and thermal insulation members. The selected area of research presented in this paper is forcing on the development of honeycomb cores for sandwich structures used in the above mentioned applications especially in places where lightweight, high toughness, vibration and acoustics damping are required. Moreover, the use of this type of structures finds the same interest in many other application areas such as ships and yacht industry and it represents state-of-the-art in materials technology [1].

Honeycomb cores that have in-plane negative Poisson's ratio (auxetic) are recently developed with different shapes and geometries [2-9]. An example of how to achieve auxetic behaviour is through modification of the geometry of hexagonal honeycomb unitcell into one in which the hexagonal cells adopt a re-entrant hexagonal shape as shown in Table (1). Re-entrant hexagonal honeycomb is auxetic when the deformation mechanism is occurred by flexing or hinging of the cell ligaments [3-5]. The advantages of the auxetic honeycombs over the traditional hexagonal positive poison's ratio honeycombs are the enhancements of many physical properties such as energy absorption capability, plane strain fracture toughness and the ability to form synclastic (dome shape) curvatures under out-of-plane bending [3, 9]. Auxetic honeycombs have recently attracted many researchers to develop new designs that can be implemented in many industrial applications for their superior properties over the conventional ones. [3-9]. Auxetic honeycomb structures have also been good candidate for the design of morphing wings [13-15] and many other aerospace structures [15-18].

Analytical expressions for the in-plane properties of the six-ligament honeycomb structures have been derived and presented by many authors [10-12]. For most cases, these expressions are based on treating the behaviour of the honeycomb ligaments as simple Euler beams. Masters and Evans [12], derived expressions for Poisson's ratios, modulus of elasticity and the shear modulus. The expressions listed in Table 1 consider combination of the three deformation mechanisms mentioned above. These expressions are valid for both conventional hexagonal honeycomb and the auxetic re-entrant honeycombs. The re-entrant effect of the hexagonal unitcell on the mechanical properties of the honeycomb is shown clearly in Fig. 1.

In the present work, the in-plane mechanical properties of the hexagonal re-entrant honeycomb structure were studied. As mentioned above as a result of the re-entrant topology of the hexagonal honeycomb, the behaviour of the honeycomb exhibits the auxetic behaviour i.e. the resulting in-plane Poisson's ratio is negative. Moreover the in-plane modulus of elasticity is reduced dramatically as shown in Fig. 1b. In an attempt to improve the in-plane modulus of elasticity and keeping the auxetic behaviour, two new designs of the unitcell are presented based on the basic re-entrant topology. 


\section{Table 1. Unitcell shape and expressions for the in-plane properties of the conventional hexagonal and the re-entrant hexagonal honeycombs}

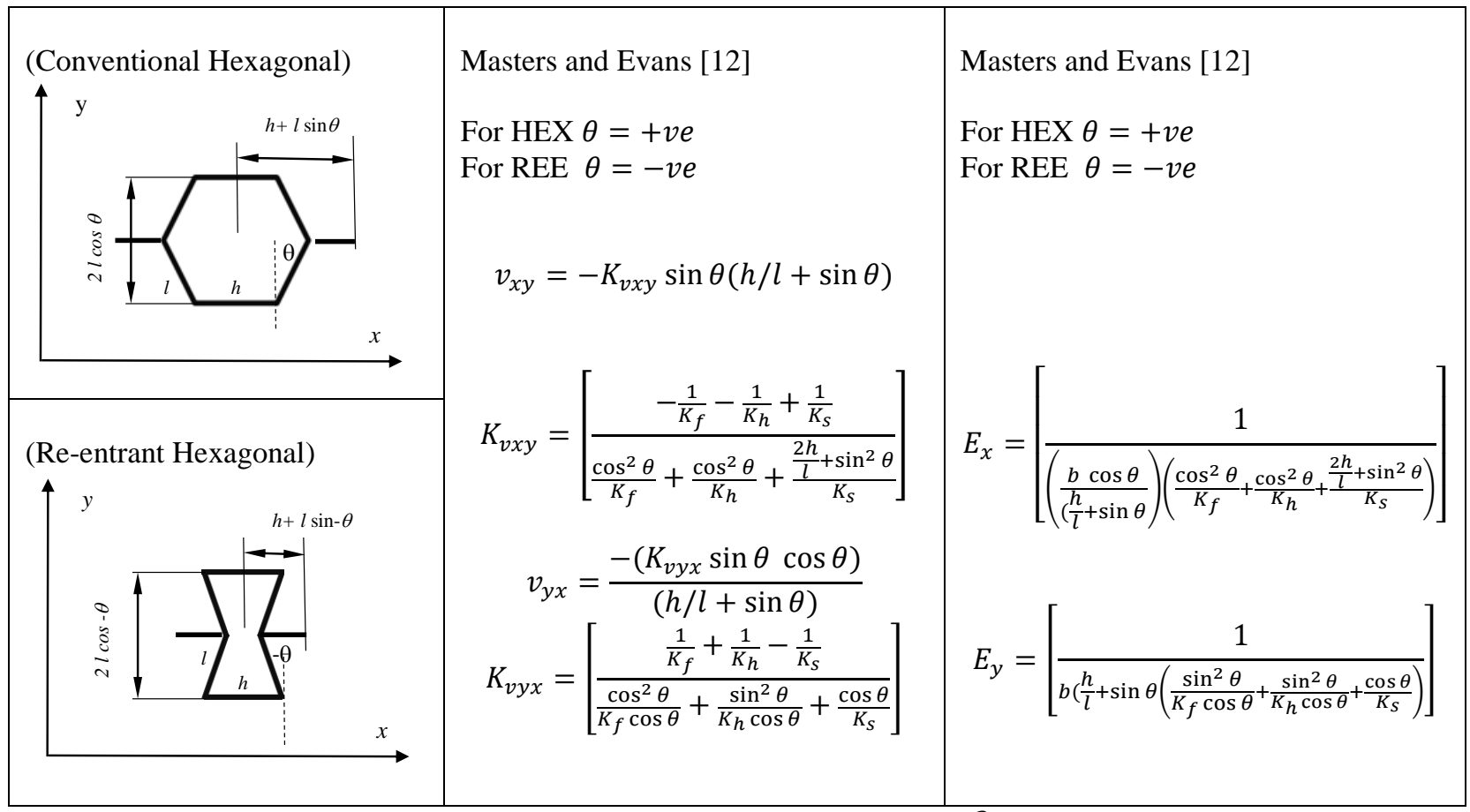

Where $b$ is the depth of the topology and $K_{l}=E_{s} b(t / l)^{3}, \quad K_{h}=\left[E_{s} b / 2\left(1+v_{s}\right)\right](t / l)$, $K_{s}=E_{s} b(t / l)$

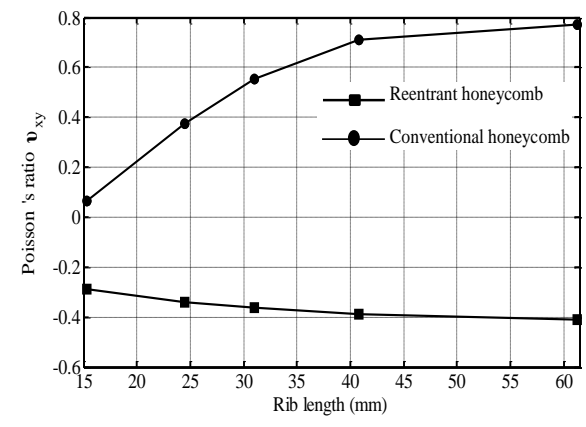

(a)
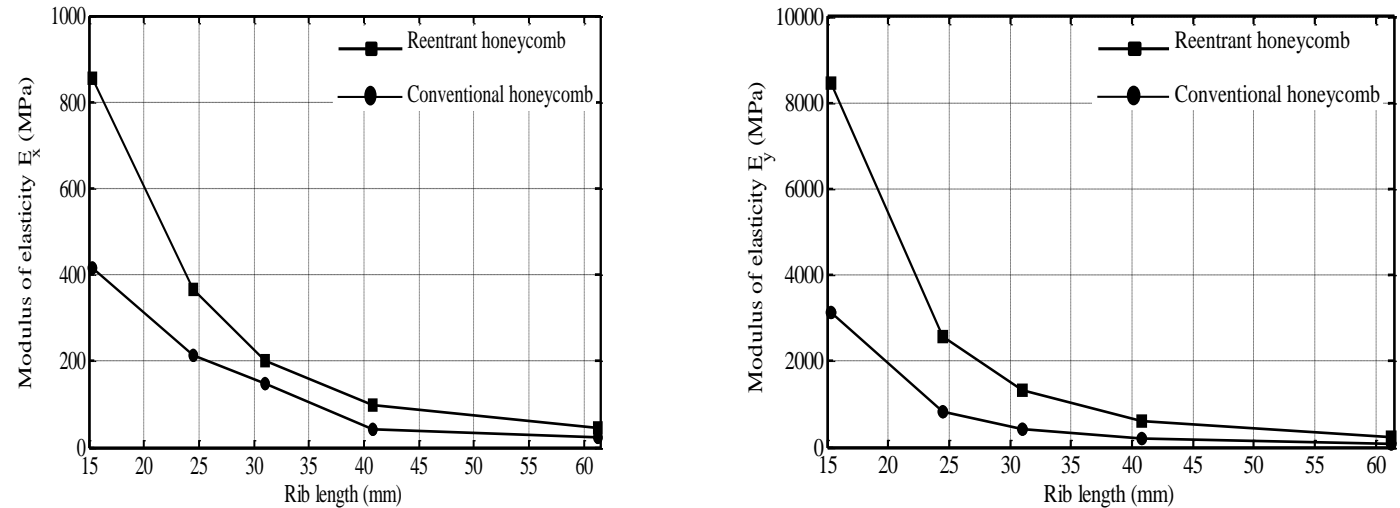

(b)

Fig. 1. Comparison between the mechanical properties of the conventional and auxetic hexagonal honeycombs (a) Poisson's ratio ( $v_{x y}$ and $\left.v_{y x}\right)$ (b) Modulus of elasticity $\left(E_{x}\right.$ and $\left.E_{y}\right)$ 


\section{Unit-cell Design Modification}

Modifications on the basic re-entrant hexagonal unitcell were introduced in two designs namely splined-reentrant and stiffened reentrant. In the splined-reentrant design, the two vertical ligaments of the re-entrant unitcell were replaced by elliptical pockets as shown in Fig. 2. The major diameter of the elliptical pocket has the same length as the length of the original ligament and the minor diameter is dependent on the ligament length and the reentrant hexagon angle. The main design criteria for the first design is to improve the in-plane buckling and stretching mechanisms of the vertical ligaments. In a similar fashion, the stiffened-reentrant, in addition to the modifications introduced in the splined-reentrant design a horizon pocket is added to link the inclined ligaments to improve the flexing stiffness of the unitcell. The dimensions of the horizontal pocket is selected to be a fraction of the geometrical parameter $\mathrm{R}$ between $0.65-0.75$ of the main vertical pockets as shown in Fig. 2 .

(a)
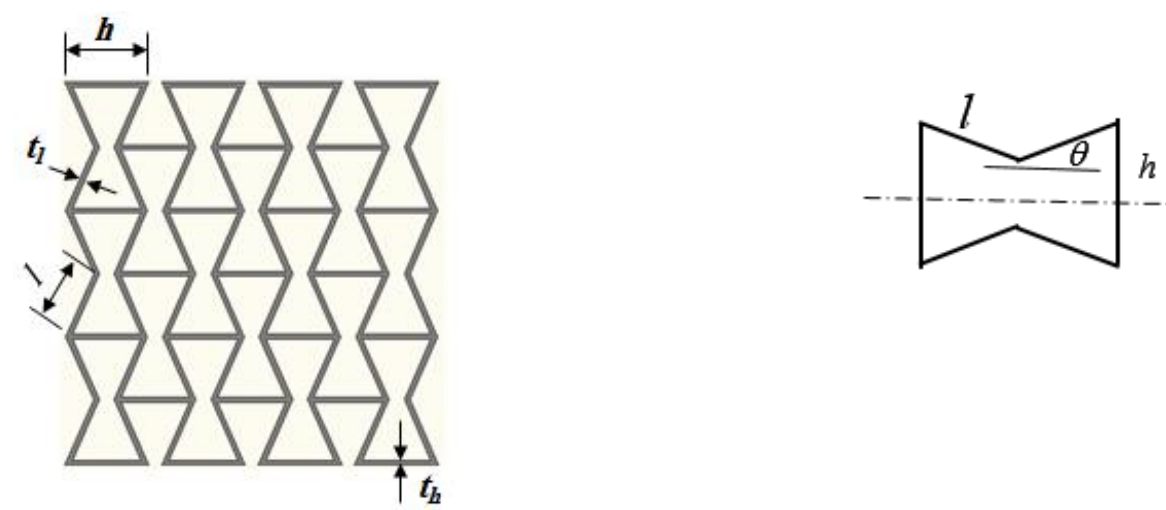

(b)
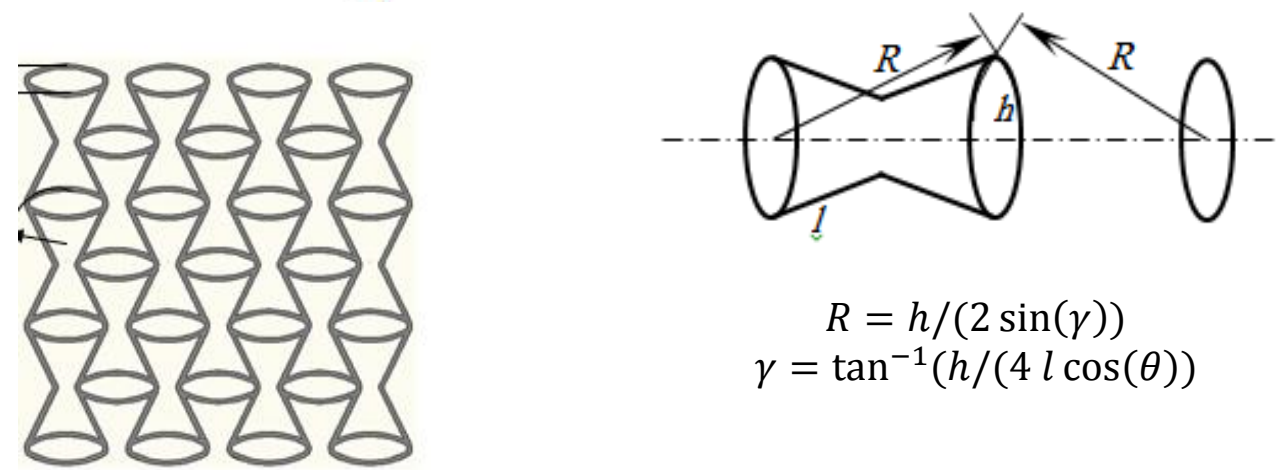

$$
\begin{gathered}
R=h /(2 \sin (\gamma)) \\
\gamma=\tan ^{-1}(h /(4 l \cos (\theta))
\end{gathered}
$$

(c)
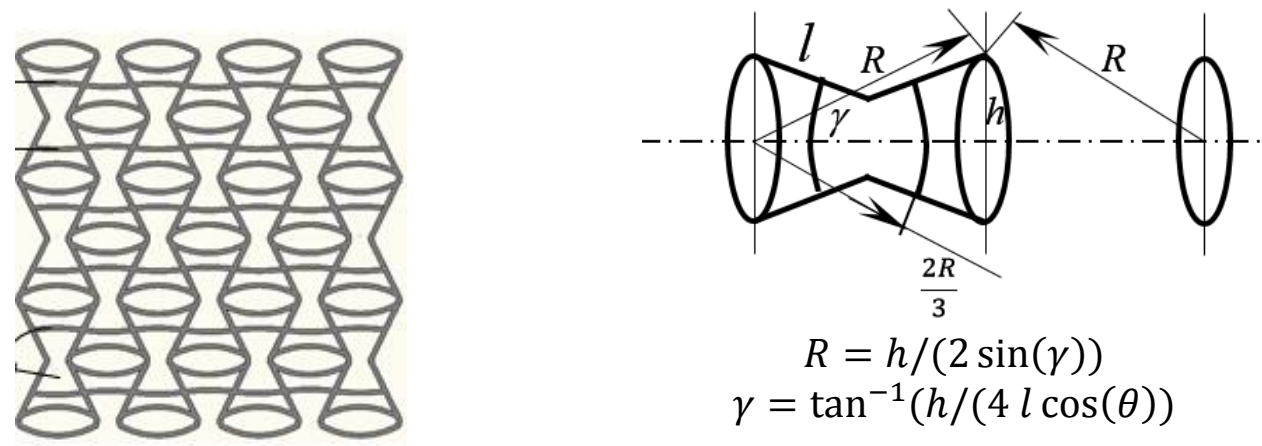

Fig. 2. Unit-cell design of (a) Re-entrant (b) Stiffened Re-entrant and (c) Splined Reentrant 


\section{Finite Element Modelling}

\subsection{FE models description}

Finite element models are constructed using ANSYS package version 12. Models are constructed first for the re-entrant structure in order to verify the FE results with those obtained previously in the literature as described in Section 3.1. Other set of models has been developed for the and for the two new designs. For all model SHELL99 element type is used to model the behaviour. The material of the auxetic network is considered to be isotropic and the properties for the solid material of the auxetic structures are taken as $E_{s}$ and $v_{s}$. Meshing for the auxetic structures is carried out using mapped meshing schemes as shown in Fig. 3. The apparent in-plane mechanical properties of the structures such as, Young's modulus and Poisson's ratios are determined in $\mathrm{x}$ and $\mathrm{y}$ directions by applying uniaxial loads. The model is formed in the shape of a rectangular shape has at least an aspect ratio of 1.5. Also, at least 25 unit cells are used to determine the mechanical properties of the structure. The honeycomb is fixed at one end and the load is applied at the other end for both $\mathrm{x}$ and $\mathrm{y}$ directions. The engineering strain of the whole combination is used to determine the apparent properties of the honeycomb. The deformed shape for the new designs and the basic reentrant structures under tension are shown in Fig. 4. It is clearly shown that the auxetic behaviour was observed for the three structures.

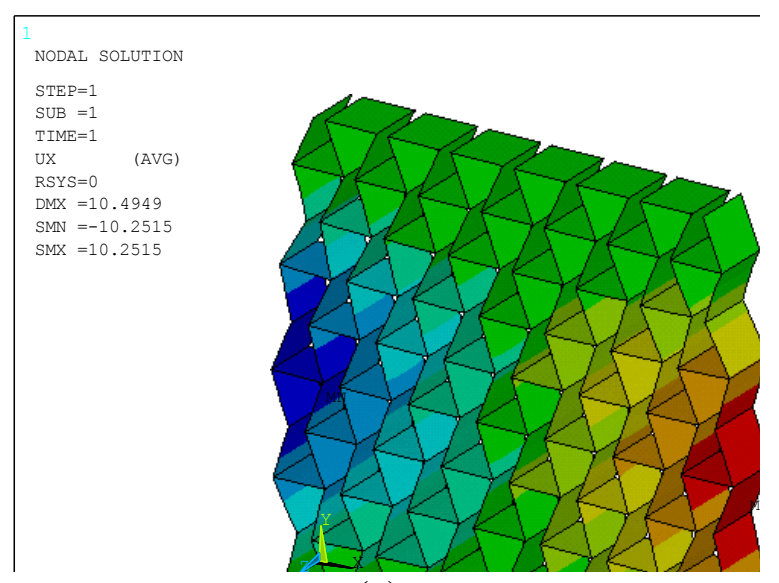

(a)

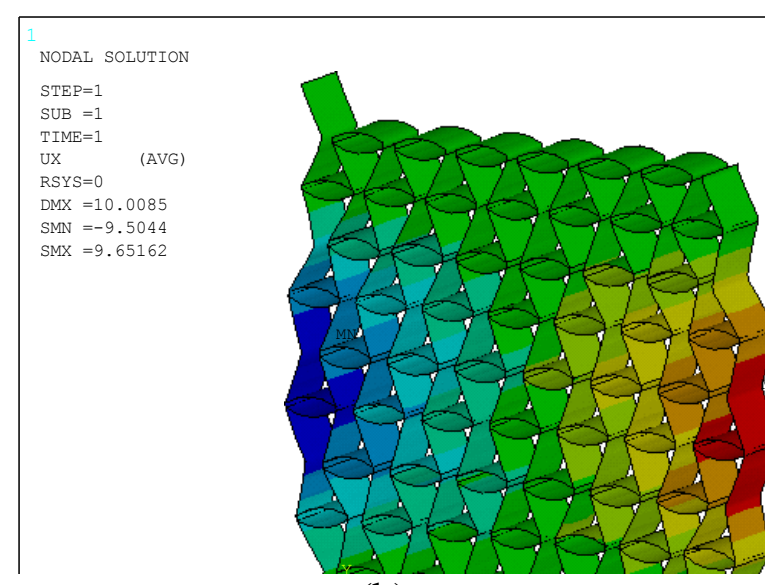

(b)

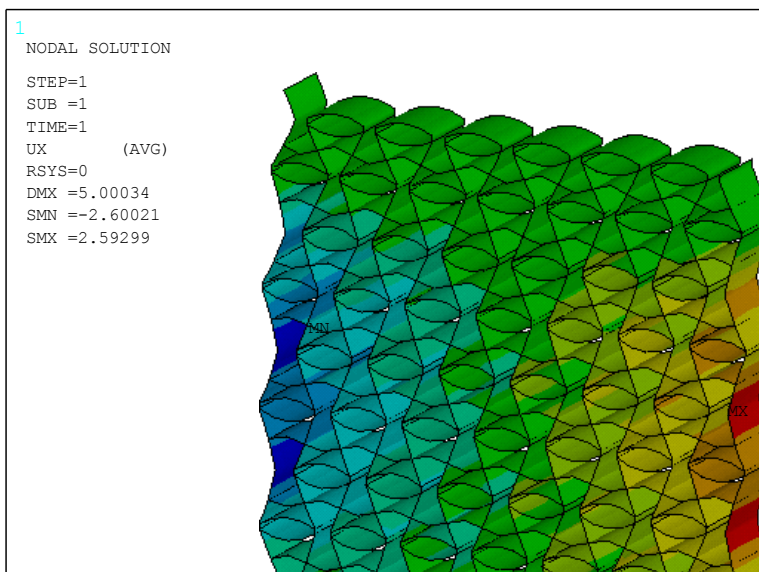

(c)

Fig. 3. The deformed shape of the structures under tension using ANSYS (a) Basic Reentrant (b) Splined-Re-entrant and (c) Stiffened Re-entrant 


\subsection{FE models verifications of the Re-entrant structure}

Finite element results of Poisson's ratios $v_{x y}, v_{y x}$ and Young's moduli $E_{x}, E_{y}$ for the reentrant structure have been verified by the analytical expressions listed in Table 1 . The elastic constants of the auxetic network are calculated using the above analytical models. The calculations are based on the assumption of the solid material constants $E_{s}=210 \mathrm{GPa}$ and $v_{s}$ $=0.30$, the rib thickness $\mathrm{t}_{\mathrm{l}}=\mathrm{t}_{\mathrm{h}}=3 \mathrm{~mm}$, the angle $\theta=-23^{\circ}$. Table 2 shows the variation of the inplane Poisson's ratio and Young's modulus of the re-entrant structure with the ligament length, $l=h$. As can be seen from Table 2 good agreement between the analytical and the finite element models has been obtained. The error percentage is less than $8 \%$ for Poisson's ratio and is less than $5 \%$ for young's modulus. This can be attributed to that, the analytical solution is based on the analysis of one unit-cell with the ligaments treated as simple beams while in the FE models the SHELL99 element is used to model in which the network is considered as a thin plate with about 25 unit-cell in the model.

Table 2. Comparison between FE models and analytical expressions results of the inplane properties of the Re-entrant honeycomb for $t_{l}=t_{h}=3 \mathrm{~mm}, \theta=-23^{0}, \mathrm{~b}=8 \mathrm{~mm}$

\begin{tabular}{|c|c|c|c|c|c|c|c|c|}
\hline \multirow{2}{*}{$\begin{array}{l}\text { Rib Length } \\
l(\mathrm{~mm})\end{array}$} & \multicolumn{2}{|c|}{$v_{x y}$} & \multicolumn{2}{|r|}{$v_{y x}$} & \multicolumn{2}{|c|}{$\begin{array}{c}E_{x} \\
(\mathrm{GPa})\end{array}$} & \multicolumn{2}{|r|}{$\begin{array}{c}E_{y} \\
(\mathrm{GPa})\end{array}$} \\
\hline & FE & Analytical & $\mathrm{FE}$ & Analytical & FE & Analytical & FE & Analytical \\
\hline 15 & -0.354 & -0.333 & -2.79 & -2.99 & 0.855 & 0.735 & 8.46 & 8.64 \\
\hline 25 & -0.342 & -0.303 & -2.93 & -3.10 & 0.207 & 0.206 & 2.55 & 2.53 \\
\hline 30 & -0.285 & --0.295 & -2.99 & -3.20 & 0.108 & 0.107 & 1.31 & 1.32 \\
\hline 40 & -0.246 & -0.289 & -3.42 & -3.46 & 0.046 & 0.049 & 0.629 & 0.640 \\
\hline 60 & -0.237 & -0.279 & -3.62 & -3.65 & 0.016 & 0.017 & 0.206 & 0.210 \\
\hline
\end{tabular}

\subsection{FE Results for the new designs}

A parametric study is carried out on the new designs to investigate the variation of the in-plane mechanical properties with the unitcell geometrical parameters namely rib (ligament) length $l$ and rib (ligament) thickness, $t$. Figs. 4, 5 shows the variation of the properties of rib length $l$ and rib thickness $t$, respectively. The results were compared with the basic re-entrant to show the improvement of the properties. The re-entrant rib angle was kept constant and the other parameter were varied which mainly reflect the effect of the relative density of the honeycombs $\rho^{*}$ [5]. The relative density is defined as the planner area of the unitcell ribs divided by the projected area of the unitcell. 

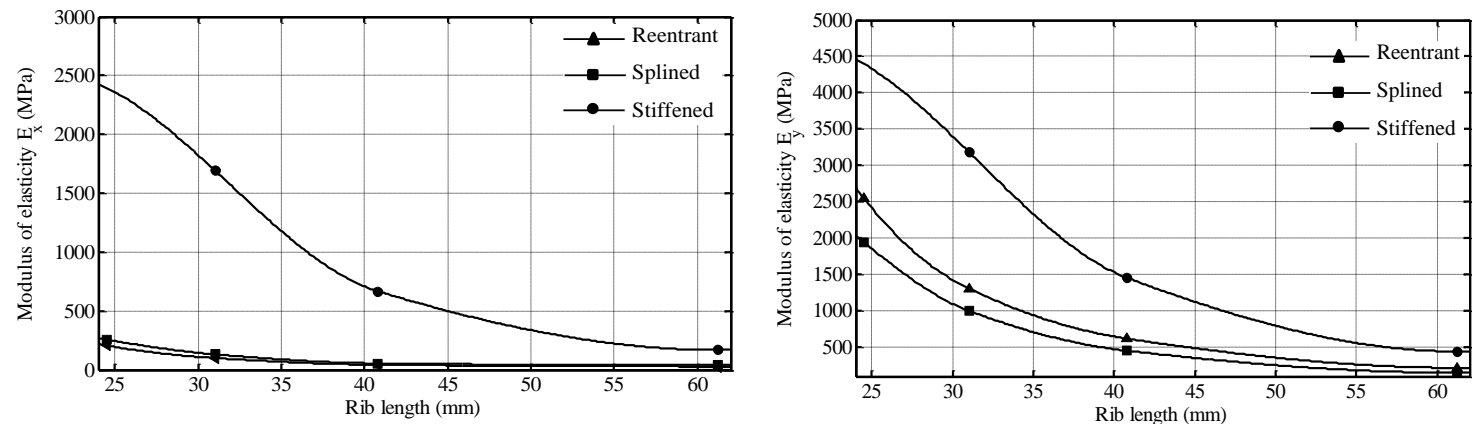

(i)
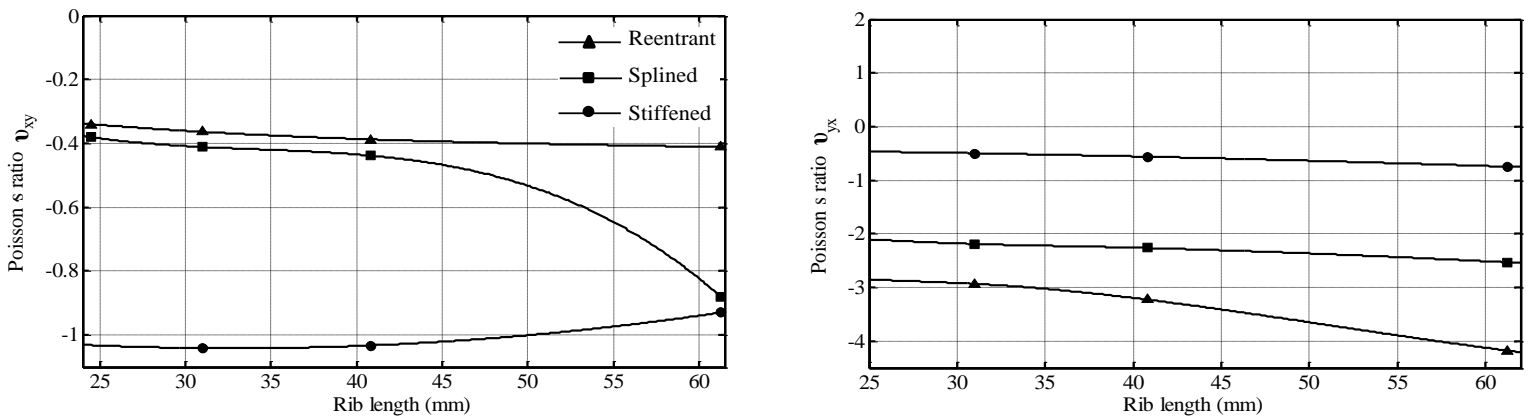

(ii)

(a)
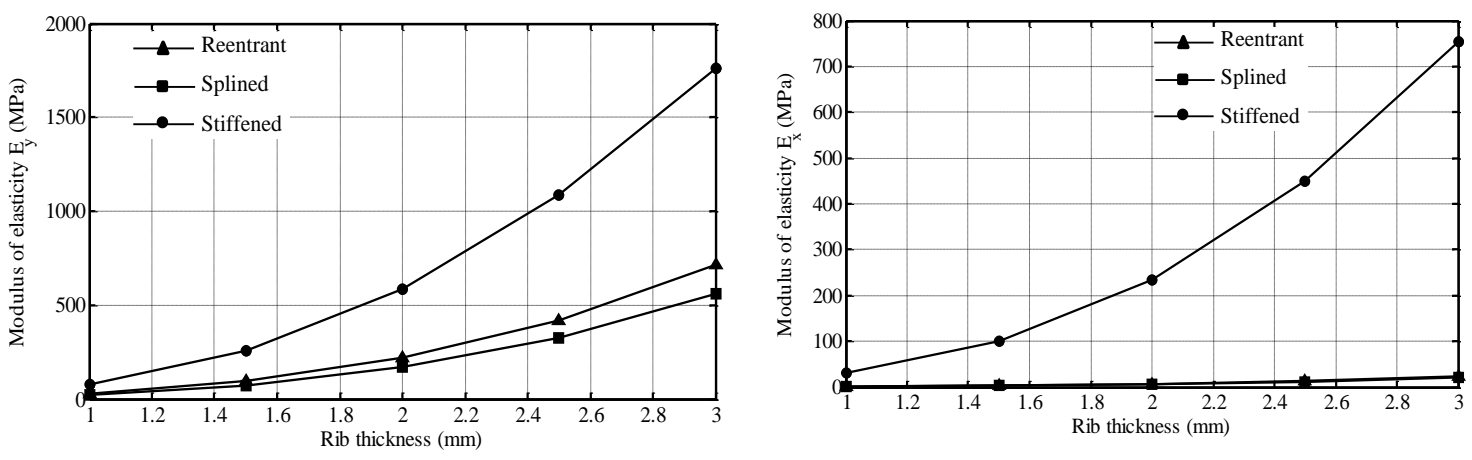

(i)
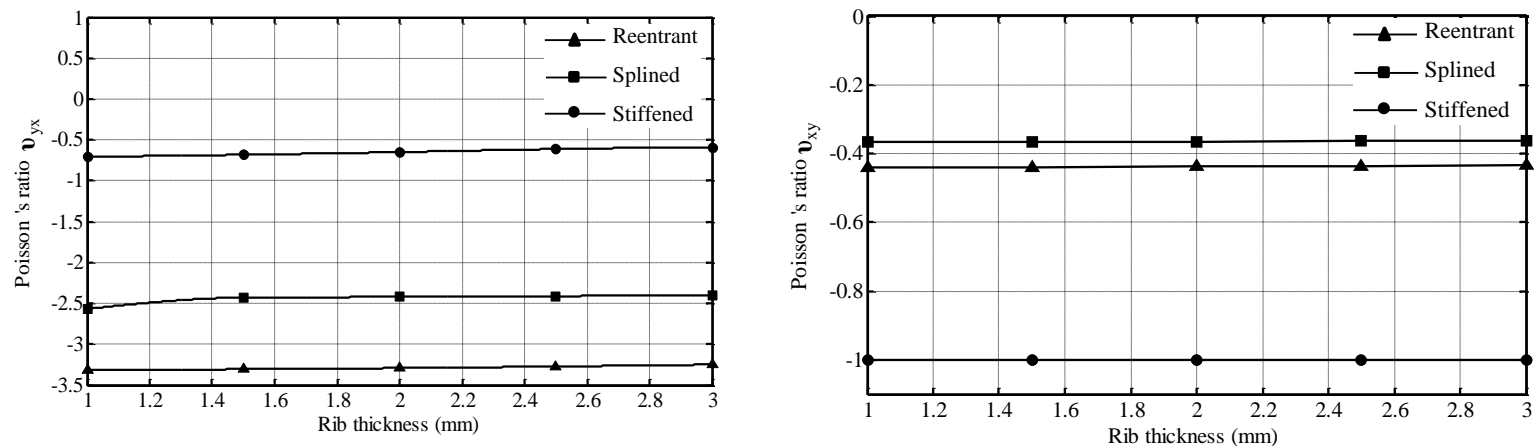

(ii)

(b)

Fig. 5. The variation of the in-plane properties of the honeycombs (i) Young's Modulus (ii) Poisson's ratio with (a) the rib length $l$, (b) the rib thickness $t$. 


\section{Experimental Work}

In order to verify the finite elements results compression test were carried out on samples of the new designs and the basic re-entrant honeycombs. Samples of the three designs were made of steel with Yung's modulus $\mathrm{E}_{\mathrm{s}}=210 \mathrm{GPa}$ and Poisson's ratio $v_{s}$ of 0.3 . The samples were fabricated to the required shape and geometry using laser cutting technique. Samples were formed in a rectangular shape with top and bottom edges attached to a solid steel bar to allow uniform loading of the honeycombs edge. A computerized universal testing machine of capacity $200 \mathrm{kN}$ is used for the compression test. The displacement rate was $0.5 \mathrm{~mm} / \mathrm{min}$ to almost a strain up to $5 \%$ to ensure that data are recorded within the elastic limit of the samples. In some tests the strains exceeded the 5\% to investigate the deformation mechanism but still within the elastic range. The load and displacement graphs were produced using the machine data acquisition system to evaluate the sample modulus. The test was repeated for both vertical and horizontal axes of the samples. The whole test was recorded using a video camera. Marks were placed at several places on the test samples to monitor the deformation of the honeycombs structure. The video data was processed using MATLAB image processing toolbox to extract deformations of the samples in different positions along the sample axes in $\mathrm{x}$ and $\mathrm{y}$ directions. The deformation data extracted from the image processing is used to measure the sample longitudinal and lateral strains in order to evaluate the structure Poisson's ratio as follows:

$$
\begin{aligned}
& \varepsilon_{\mathrm{x}}=\left(\mathrm{x}_{\mathrm{n}}-\mathrm{x}_{\mathrm{o}}\right) / \mathrm{x}_{\mathrm{o}} \\
& \varepsilon_{\mathrm{y}}=\left(\mathrm{y}_{\mathrm{n}}-\mathrm{y}_{\mathrm{o}}\right) / \mathrm{y}_{\mathrm{o}}
\end{aligned}
$$

Where $x_{n}$ is the distance between marks at the nth step of loading. $x_{o}$ and $y_{o}$ are the sample lengths before loading. The average strain for the structures was calculated by taking the average of the strains determined from different locations of the marks, and then the Poison's ratio was calculated from the average strains as follows:

$$
v_{x y}=-\varepsilon_{\mathrm{y}} / \varepsilon_{\mathrm{x}}
$$

The tensile strength $\sigma$ can be calculated from the following equation:

$$
\sigma_{x}=\frac{F_{x}}{A_{x}} \text { and } \sigma_{y}=\frac{F_{y}}{A_{y}}
$$

where;

$F$ : The applied load in elastic range in $\mathrm{x}$ or $\mathrm{y}$ directions $(\mathrm{N})$.

$A$ : The projected cross-section area of test sample $\mathrm{A}_{\mathrm{x}}=b \mathrm{x}_{\mathrm{o}}$ and $\mathrm{A}_{\mathrm{y}}=b \mathrm{y}_{\mathrm{o}}\left(\mathrm{mm}^{2}\right)$.

The average value of Poisson's ratio $\left(v_{y x}\right)$ and modulus of elasticity $\left(E_{y}\right)$ from the slopes of the apparent transverse strain vs. apparent axial strain and the axial stressstrain curves. Fig. 6 and Fig. 7 show the load-displacement curves and samples of the three designs loaded in the longitudinal and lateral directions respectively. 

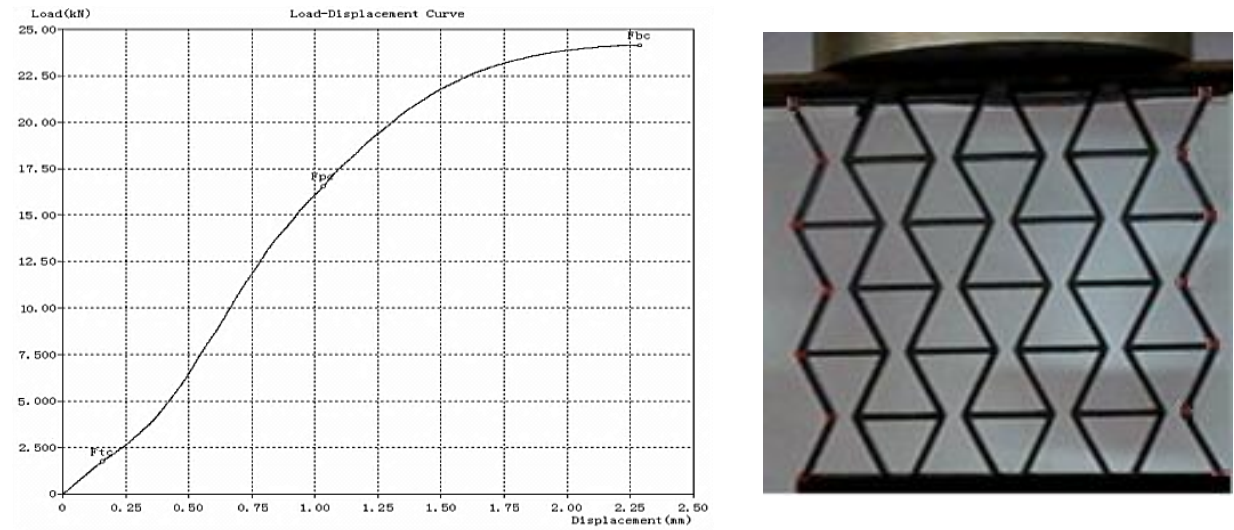

(a)
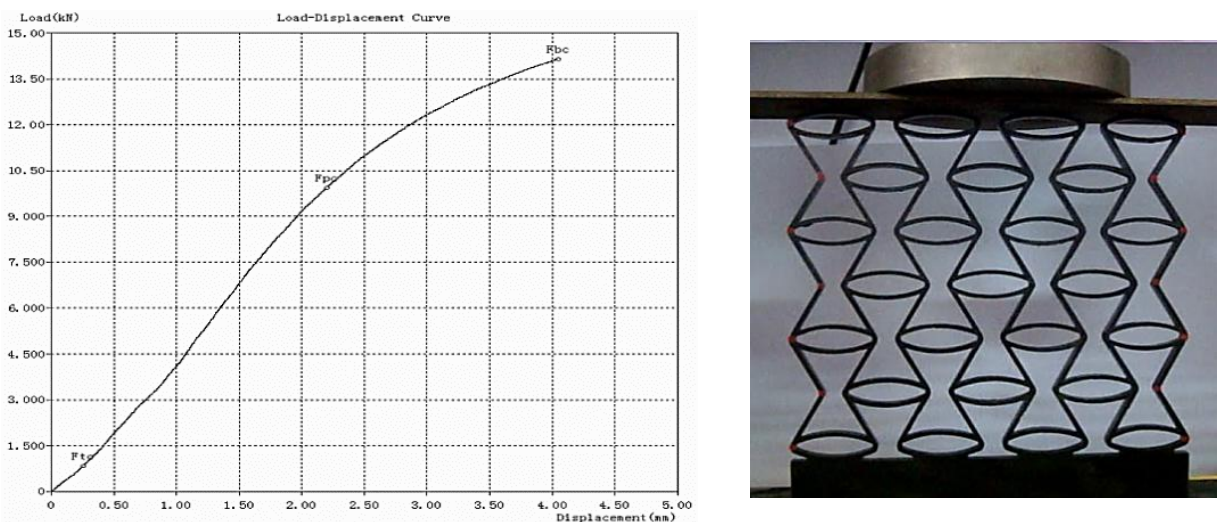

(b)


(c)

Fig. 6. F- $\delta$ curves in longitudinal for (a) Re-entrant (b) Splined-reentrant and (c) Stiffened re-entrant honeycombs 

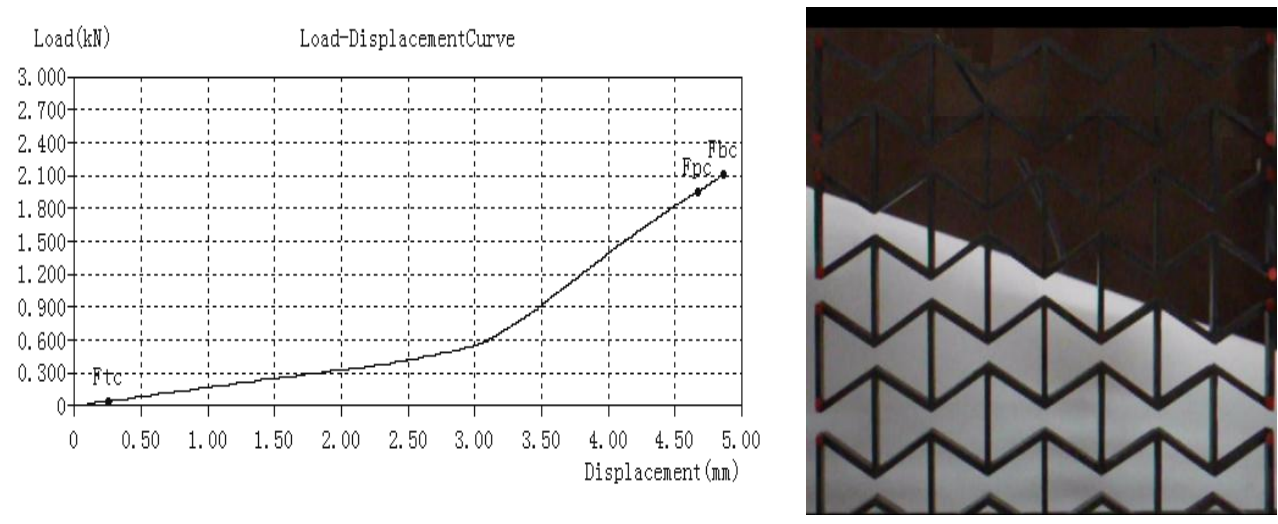

(a)
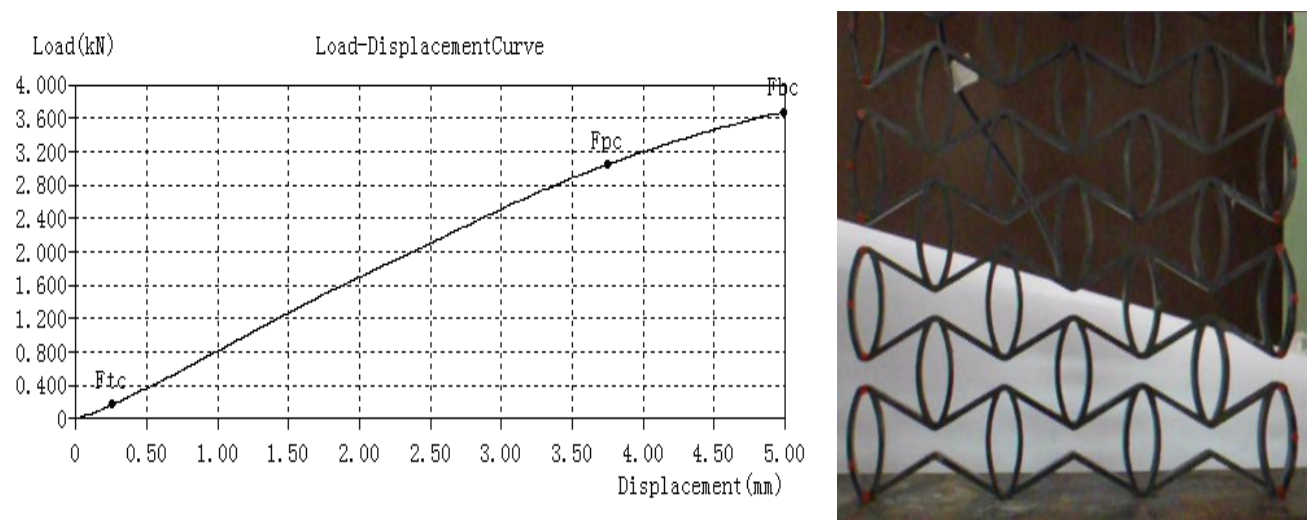

(b)
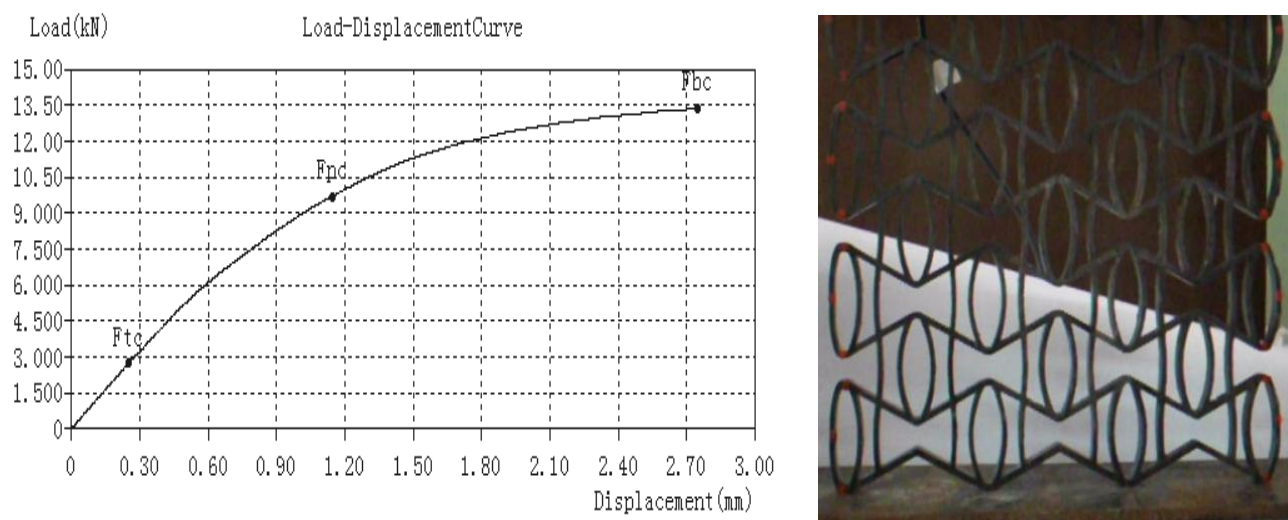

(c)

Fig. 7. F- $\delta$ curves in longitudinal for (a) Re-entrant (b) Splined-reentrant and (c) Stiffened re-entrant honeycombs 


\section{Results and Discussion}

The results obtained by the finite elements method are illustrated in Figs. 4, 5 which show the variation of the rib dimensions of the unitcell for constant rib angles. The results are presented for the three designs for the purpose of comparison. It is clear from these figures that the auxetic behaviour is still dominating the deformation of the new designs. However the values of the negative Poisson's ratio in both directions of the honeycomb are varying in a fashion to keep the same bulk modulus of the whole structure. For example the in-plane properties of a honeycomb with the dimensions $l=h=30 \mathrm{~mm}$ and $t_{\mathrm{h}}=\mathrm{t}_{1}=3 \mathrm{~mm}$ and rib angle of $-23^{\circ}$ and takes the stiffened-reentrant shape were increased with the ratio $\left(E_{x}=16.6\right.$ times, $E_{y}=2.4$ times, $\mathrm{v}_{\mathrm{xy}}=$ negativity increased 3 times and $\mathrm{v}_{\mathrm{yx}}=$ negativity decreased 3 times) compared to the honeycomb takes the basic re-entrant shape. This can be attributed to the increase of the structure relative density due to the addition of the horizontal and vertical pockets to the basic design. Also from the same figures as the rib ligament increases the modulus of the structure in the longitudinal and the lateral direction decreases for the three structures this can be attributed to that the relative density of the unitcell decreases dramatically. However the values of Poisson's ratios were kept constant for a certain rib length in which afterwards it starts to go near more negativity due to the increase of the rib slenderness ratio. The increase of the rib slenderness ratio causes that the dominating deformation mechanism is due to flexing of the inclined ribs. In contrary the increase of the rib thickness improves the in-plane stiffness due to the increase of the relative density of the unitcell but with almost no significant change in the Poisson's ratio.

Compression tests were carried out on honeycomb samples made of steel using laser cutting technique to verify the FE results obtained for the new designs. It was noted that during the test the auxetic samples do not exhibit the well-known plateau shape of the load-deformation curve of the conventional honeycomb which is attributed to the re-entrant effect of the unitcell and conform with the explanation described by Gibson and Ashby [3]. Also, from the loaddisplacement diagrams for the three designs it was noted that the slope of the curve during the elastic zone was changed to a different slope than the initial slope which indicate the elastic buckling of the unitcell ribs and confirms the deformation mechanism take place for the reentrant shape of the unitcell. A comparison between the finite element results and the compression test results is listed in Table 3. The results were in good agreement however the experimental values were found to be higher than the finite elements results. The percentage difference is about $2 \%$ for the moduli and about $13 \%$ for Poisson's ratio. The error for Poisson's ratio can be attributed to the use of image processing for measuring the deformation as the images resolution may affect the values of the local deformation on the test sample.

Table 3. Comparison of the in-plane properties of the honeycombs designs obtained experimentally and using FE method for $l=h=42 \mathrm{~mm}, t_{h=} t_{l}=2.86$ and $\theta=-23^{o}$

\begin{tabular}{c|c|ccc}
\hline \multicolumn{2}{c|}{ Parameters } & Re- & Splined- & Stiffened- \\
\hline Modulus of elasticity $E_{x}$ & FEM & 42.4 & 55.23 & 725.54 \\
$(\mathrm{MPa})$ & Experimental & 44.829 & 53.97 & 715.12 \\
Modulus of elasticity $E_{y}$ & FEM & 618.54 & 471.43 & 1343.93 \\
$(\mathrm{MPa})$ & Experimental & 623.8 & 467.67 & 1357.62 \\
Poisson's ratio $v_{y x}$ & FEM & -3.23 & -2.34 & -0.53 \\
& Experimental & -3.59 & -2.65 & -0.61 \\
Poisson's ratio $v_{x y}$ & FEM & -0.39 & -0.45 & -1.06 \\
& Experimental & -0.32 & -0.39 & -0.999 \\
\hline
\end{tabular}




\section{Conclusions}

The two new designs presented in this work showed a significant improvement of in-plane stiffness of the honeycomb cores. The auxetic behaviour of the original basic structure was kept functional but with different level of auxeticity. The stiffened-reentrant structure showed better enhancement in both of the moduli of the structures in the axial and the lateral directions. A parametric study on varying the structures unitcell dimensions using the finite elements method was carried out on the three structures. The parametric study showed the significant effect of the rib length on the in-plane moduli of the structures. It is concluded that the increase of the rib length reduces dramatically the in-plane relative modulus which in turns reduces the Young's moduli of the structures. The increase of the rib thickness contributes to the increase of the structures moduli with slight effect on Poisson's ratios. Compression tests were carried on honeycomb samples made of steel using laser cutting technique with different geometrical parameters. Test results for the three designs were compared with the finite element results and they were in a good agreement.

\section{References}

[1] Bitzer, T (1997). Honeycomb Technology: Materials, Design, Manufacturing, Applications and Testing, London: Chapman \& Hall.

[2] A. Alderson, K. L Alderson, 2007. „Auxetic materials“ Proc. ImechE, 221 Part G ; 1 Aerospace Engineering, pp565-575.

[3] Gibson LJ, Ashby MF. Cellular solids, structure and properties. $2^{\text {nd }}$ ed. Cambridge: Cambridge University Press; 1997.

[4] D, Lakes R. Properties of a chiral honeycomb with Poisson's ratio -1. Int. J. Mech. Sci. 1996;39:305314.

[5] Lakes R S, 1987, Foam structures with a negative Poisson's ratio Science 2531038 -40.

[6] Evans K 1991 The design of doubly curved sandwich panels with honeycomb cores Compos. Struct. 17 95-111.

[7] A. Lorato, P. Innocenti, F. Scarpa, N. Ravirala, K M Zied, A. Alderson, K. Alderson, W Miller, C. W Smith, K. E Evans. "The transverse elastic properties of chiral honeycombs". Journal of Composites Science and Technology. doi:10.1016/j.compscitech.2009.07.008.

[8] A. Alderson, K. L Alderson, D. Attard, K. E Evans, P. S Farrugia, R. Gatt, J. N Grima W. Miller, N. Ravirala, C. W Smith, K. M Zied. 2009."Elastic constants of 3-, 4- and 6-connected chiral and anti-chiral honeycombs subject to uniaxial in-plane loading". Journal of Composites Science and Technology. doi:10.1016/j.compscitech.2009.07.009.

[9] A. Alderson, K. L. Alderson, N. Ravirala, K.M. Zied. The in-plane linear elastic constants and out of plane bending of 3-coordinated ligament and cylinder-ligament honeycombs" Journal of Composites Science and Technology. doi:10.1016/j.compscitech.2009.07.010.

[10] J. Whitty, F. Nazare, A. Alderson. "Modelling the effect of density variations on the in-plane Poisson's ratios and Young's modui of periodic conventional and re-entrant honeycombs- Part 1: rib thickness variation." Cellular Polymers 21(2), pp69-97, 2005.

[11] Evans K E 1991 Auxetic polymers: a new range of materials Endeav. New Ser. 15 170-4

[12] Masters I and Evans K 1996 Models for the elastic deformation of honeycombs Compos. Struct. 35 403-22 
[13] C.1. Thill, J. Etches, I. Bond, K. Potter, and P. Weaver, 2008, "Morphing Skins," The Aeronautical Journal, No. 3216.

[14] Martin J, Heyder-Bruckner J J, Remillat C, Scarpa F, Potter K and Ruzzene M 2008 The hexachiral prismatic wingbox concept Phys. Status Solidi b 245 570-7

[15] Spadoni A, Ruzzene M and Scarpa F 2006 Dynamic response of chiral truss-core assemblies J. Intell. Mater. Syst. Struct. 17 941-52

[16] Bornengo D, Scarpa F and Remillat C 2005 Evaluation of hexagonal chiral structure for morphing airfoil concept Proc. Inst. Mech. Eng. G 219 $185-92$

[17] Scarpa F, Smith F C, Chambers B and Burriesci G 2003 Mechanical and electromagnetic behaviour of auxetic honeycomb structures Aeronaut. J. 107 175-83.

[18] Whitty J.P.M., Alderson A., Myler P., Kandola B., Towards the design of sandwich panel composites with enhanced mechanical and thermal properties by variation of the in-plane Poisson's ratios. Composites. Part :Appl. Science and Manufacturing, 2003, 34, 525-534. 